Ann. Génét. Sél. anim., 1975, 7 (2), I9I-196.

\title{
TAILLE DE LA CRETE OU DES BARBILLONS DES COQUELETS A 10 SEMAINES COMME INDICE DE MATURITÉ SEXUELLE DANS LA SÉLECTION POUR LA PONTE
}

\author{
H. AYOUB et P. MÉRAT* \\ avec la collaboration technique de L. Durand* \\ Faculté d'Agriculture, Université Ain-Shams, \\ Le Caire (Égypte) \\ * Laboratoire de Génétique factorielle, \\ Centre national de Recherches zootechniques, I. N. R. A., \\ 78350 Jouy en Josas \\ RÉSUMÉ
}

Dans notre population, la longueur moyenne des barbillons des frères à ro semaines présente une corrélation négative appréciable avec l'âge au rer œuf de leurs sœurs, et une légère corrélation positive avec leur ponte d'automne et d'hiver. Les corrélations génétiques apparaissent de même signe, et l'héritabilité de la longueur des barbillons est relativement élevée. Ceci suggère la possibilité d'utiliser ce caractère comme critère individuel de sélection des coquelets pour leur potentiel génétique concernant l'âge de la maturité sexuelle.

\section{INTRODUCTION $(\mathbf{1})$}

Le développement de la crête et des barbillons, en tant que caractères sexuels secondaires chez la Poule, peut représenter chez les jeunes un indice simple de maturité sexuelle.

PASVOGEL et al. (I95I), PASVOGEL (I952) trouvent une corrélation positive entre ponte des poules et taille de la crête de leurs frères ou de leurs pères à II semaines.

(1) Ce travail est contenu en partie dans une thèse de Docteur-Ingénieur (H. AyouB, r97I). 
Ils proposent l'utilisation de ce critère pour aider à la sélection des mâles sur la ponte. GAI,PERN et BongaIEv (Ig66) semblent proposer une conclusion analogue.

On peut peut-être citer dans le même ordre d'idées les résultats de GoodwiN et al. (1955) qui comparent trois lignées de Leghorns sur plusieurs années. Chez l'une d'elles, relativement infertile, les femelles entrent en ponte une semaine plus tôt que dans les autres en moyenne. Ceci s'accompagne d'une taille plus grande de la crête, de l'hypophyse, et des testicules chez les mâles, l'ensemble traduisant sans doute une différence d'activité gonadotrop?.

Nous avons voulu chercher plus spécialement ici si la taille des barbillons des coquelets à ro semaines est utilisable comme indice de leur potentiel génétique pour la précocité de ponte.

\section{MATÉRIEL ETT MÉTHODES}

Trois années de la population pedigree élevée à Jouy en Josas et éclose au printemps (MERAT, 1967) sont utilisées pour cette analyse : 1966 ( 8 pères et 26 mères, chaque mère n'étant accouplée qu'à un père), I 967 (I 4 pères et 44 mères), I 969 (I 4 pères et 4 I mères). Cette population est élevée au sol, avec toutes les familles réparties dans les mêmes locaux. Elle est issue du croisement en I954 de plusieurs races de format moyen (notamment Rhode-Island et Gâtinaise), contient divers facteurs mendéliens connus en ségrégation, et n'est soumise qu'à un minimum de sélection en ce qui concerne les caractères économiques.

Les caractères étudiés sont :

- longueur des barbillons des mâles à l'âge de ro semaines,

- âge au I er œuf des poulettes,

— nombre d'œufs pondus par les poulettes (jusqu'au 3 I décembre).

Seules sont prises en considération les familles de frères-sœurs ayant plusieurs descendants de chaque sexe.

Chez les coquelets, nous avons choisi de mesurer la longueur des barbillons plutôt que la taille de la crête, car une partie des animaux possédaient le gène $R$ (crête en rose) et n'étaient pas comparables aux autres pour les mesures de la crête. De plus, nous avons éliminé de notre analyse les mâles à crête en pois, le gène $P$ réduisant en moyenne la longueur des barbillons (MÉRAT, données non publiées). La distance mesurée, pour des raisons de commodité, est celle entre la commissure du bec et l'extrémité opposée du barbillon. Une mesure unique est prise par animal.

L'analyse des résultats comprend :

I. L'estimation des corrélations phénotypiques entre moyenne des frères pour la longueur des barbillons et moyenne des sœurs pour l'âge au I er œuf ou le nombre d'œufs ; une estimation parallèle est faite par familles de demi-frères et demi-sœurs. Les corrélations partielles correspondantes, à poids à 8 semaines des mâles fixés, ont été calculées, ainsi que celle de la longueur des barbillons des mâles avec la ponte des sœurs à âge au i er œuf fixé pour ces dernières.

2. La détermination de l'héritabilité de chaque caractère mentionné, par estimation des corrélations intra-classe entre frères-sœurs et demi-frères, demi-sœurs, en tenant compte des nombres inégaux dans les sous-classes (KEMPTHORNE, I957).

3. Celle des corrélations génétiques entre caractères révélés chez les $\delta$ đo ${ }^{\star}$ et chez les 우우, selon la méthode utilisée par KINNEY et SHOFFnER (1965).

\section{RÉSULTATS}

\section{I. - Valeurs moyennes}

Les valeurs moyennes, par caractère et par année, sont données dans le tableau $\mathbf{r}$. 
TABLEAU I

Valeurs moyennes par caractère et par année

\begin{tabular}{|c|c|c|c|c|}
\hline \multirow[b]{2}{*}{ Année } & \multicolumn{4}{|c|}{ Caractère } \\
\hline & $\begin{array}{l}\text { Longueur } \\
\text { des barbillons } \\
\text { à } 10 \text { sem. } \\
\text { des mâles } \\
(\mathrm{mm})\end{array}$ & $\begin{array}{l}\text { Poids à } 8 \text { sem. } \\
\text { des mâles } \\
(\mathrm{g})\end{array}$ & $\begin{array}{c}\text { Age } \\
\text { au } 1^{\text {er }} œ u f \\
\text { (jours) }\end{array}$ & $\begin{array}{l}\text { Nombre } \\
\text { d'œufs }\end{array}$ \\
\hline 66 & 23,2 & 871 & 164,7 & 56,5 \\
\hline 67 & 20,1 & 811 & 165,2 & 63,7 \\
\hline 69 & 19,8 & 950 & 143,6 & 91,8 \\
\hline
\end{tabular}

\section{2. - Corrélations phénotypiques}

En l'absence d'hétérogénéité significative entre années, nous ne présentons que les résultats sur le total des 3 années (estimations intra-années regroupées).

Ie tableau 2 montre, par familles de frères-sœurs et par famille de demi-frères et demi-sœurs, les corrélations phénotypiques observées entre longueur moyenne des barbillons des mâles et caractères de ponte de leurs sœurs. La corrélation entre la longueur moyenne des barbillons des mâles et leur poids moyen à 8 semaines par familles de frères ou demi-frères est également mentionnée.

\section{TABLEAU 2}

Corrélations phénotypiques entre longueur des barbillons des mâles à 10 semaincs et d'autres caractères quantitatifs

(estimations intra-années regroupées)

Corrélation simple:

- par familles de frères-sœurs .........

- par familles de $1 / 2$ frères-1/2 sœurs ...

Corrélation partielle :

à poids à 8 semaines fixé pour les $\delta \hat{\sigma}$

(familles de frères-sœurs) $\ldots \ldots \ldots \ldots \ldots \ldots$

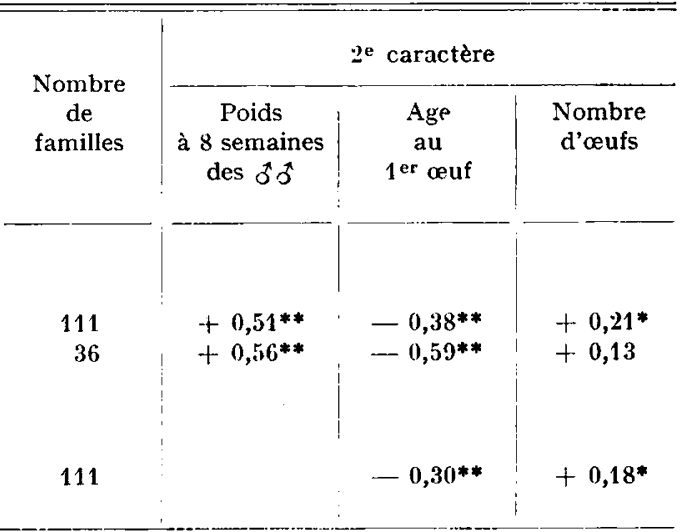

* Significatif au seuil de 5 p. 100.

** Significatif au seuil de 1 p. 100. 
Pour les deux types de familles, les résultats sont très parallèles. On vérifie d'abord que la corrélation entre poids à 8 semaines et développement des barbillons des mâles est positive et hautement significative.

Avec l'âge au premier œuf des sœurs ou demi-sœurs, la longueur des barbillons des mâles à Io semaines est en corrélation négative et très significative. I,es coquelets aux barbillons les plus développés ont les sœurs les plus précoces. En d'autres termes, le développement des caractères sexuels secondaires des mâles dès cet âge est un indice valable de précocité sexuelle.

Avec le nombre d'œufs, la corrélation, quoiqu'assez faible, est toujours positive, et significativement différente de zéro sur l'ensemble des années, au moins pour les familles de frères-sœurs : Les familles où les mâles ont les caractères sexuels les plus précoces ont une ponte moyenne légèrement supérieure. Cependant, ceci paraît traduire simplement la différence dans les âges d'entrée en ponte. En effet, par familles de frères-sœurs, le coefficient de corrélation partielle (estimé sur les années groupées) entre longueur des barbillons des mâles à ro semaines et nombre moyen d'œufs des sœurs est égal à $+0,02$ (non significatif).

Enfin, par familles de mères, le tableau 2 indique les corrélations partielles entre longueur moyenne des barbillons des frères à Io semaines d'une part, âge au I $\mathbf{I}^{\mathrm{er}}$ œuf et nombre d'œufs moyen des sœurs de l'autre, à poids à 8 semaines fixé pour les mâles. On constate que le signe et la signification de ces corrélations partielles s'accordent avec ceux des corrélations directes : Le développement des barbillons des mâles est un indice possible pour la sélection des caractères de ponte, même indépendamment de leur poids.

\section{3. - Héritabilités}

Le tableau 3 indique les estimations d'héritabilité obtenues pour chaque caractère.

\section{TABLEAU 3}

Héritabilité de la longueur des barbillons des mâles à 10 semaines et des caractères en corrélation avec elle

(années groupées)

\begin{tabular}{|c|c|c|c|c|}
\hline Caractère & $\mathbf{N}$ & $h_{\mathrm{S}}^{2}\left({ }^{1}\right)$ & $h_{\mathbf{D}}^{2}$ & $h^{2}$ \\
\hline Longueur des barbillons ( 10 sem.) .. & 562 & $0,31 \pm 0,26$ & $0,96 \pm 0,18$ & 0,64 \\
\hline Poids à 8 sem. des $\delta^{\star} \sigma \ldots \ldots \ldots$ & 562 & $0,78 \pm 0,40$ & $0,61 \pm 0,18$ & 0,69 \\
\hline Age au $1^{\text {er }}$ œuf $\ldots \ldots \ldots \ldots \ldots$ & 691 & $0,15 \pm 0,17$ & $0,57 \pm 0,14$ & 0,36 \\
\hline Nombre d'œufs $\ldots \ldots \ldots \ldots \ldots \ldots$. & 691 & $0,24 \pm 0,20$ & $0,70 \pm 0,13$ & 0,47 \\
\hline
\end{tabular}

$\mathrm{N}=$ nombre de descendants ;

$h_{\mathrm{S}}^{2}=$ héritabilité basée sur la composante "père " de la variance ;

$h_{\mathrm{D}}^{2}=$ héritabilité basée sur la composante " mère ";

$h^{2}=$ héritabilité basée sur la moyenne des deux.

(1) Intervalle de confiance approximatif, calculé sur la base d'effectifs égaux par familles. 
Les estimations pour les deux caractères de ponte sont supérieures à l'ordre de grandeur couramment trouvé, ce qui peut correspondre au fait qu'il s'agit d'une population expérimentale non soumise à une forte sélection.

Pour la longueur des barbillons dont nous n'avons pas trouvé d'estimation d'héritabilité dans la littérature, les valeurs observées sont assez élevées, notamment celle basée sur la composante "mère ", ce qui peut correspondre à des effets de dominance, d'interaction entre gènes non allèles, ou à des effets maternels.

\section{4. - Corrélations génétiques entre longueur des barbillons des coquelets et caractères de ponte}

L'estimation de ces corrélations génétiques est faite à partir de la variable " moyenne de familles de frères-sœurs ", ce qui permet d'obtenir seulement les composantes de la variance et covariance dues au père, d'où

$$
r_{\mathrm{G}}=\frac{\operatorname{Cov}_{8} x y}{\sigma_{s \cdot x} \cdot \sigma_{8 \cdot y}}
$$

(1'indice $s$ indiquant qu'il s'agit de la composante " père " de la covariance ou des variances).

En groupant les années la valeur de la corrélation génétique estimée ainsi est - 0,53 avec l'âge au I ${ }^{\text {er }}$ œuf (négative les trois années). Quant à celle avec le nombre d'œufs, il est difficile d'en tirer une conclusion, son signe variant d'une année à l'autre et sa valeur absolue dépassant 1'unité en I967.

\section{CONCLUSION}

Il apparaît donc que, dans nos conditions et pour la population analysée, le développement de la crête et des barbillons des mâles à l'âge de ro semaines est un indice précoce de la maturité sexuelle exprimée dans l'autre sexe, en liaison génétique avec elle. Il peut donc être une aide dans la sélection pour la ponte, son héritabilité assez élevée pouvant permettre une sélection individuelle d'une certaine efficacité.

On peut noter au surplus que cette sélection ne risque pas d'entraîner une réponse liée défavorable sur le poids des poules adultes. Nous observons en effet, entre la longueur des barbillons des coquelets à Io semaines et leur propre poids adulte, un coefficient de corrélation non significatif égal à - 0,02. D'autre part, indépendamment du présent travail, nous avons trouvé, entre la longueur des barbillons à ro semaines des coqs reproducteurs et le poids adulte moyen de leurs filles, sur 7 années, une corrélation non significative de $+0, I_{3}$ (regroupée intra-années, avec 76 d.1.).

Comme ceux de PASvoger, (I95I, I952), nos résultats font ressortir une liaison entre la précocité du développement des caractères sexuels secondaires des coquelets et l'âge du début de ponte de leurs sœurs ; par contre ils n'indiquent pas de corrélation avec le taux de ponte ultérieur. 


\section{REMERCIEMENTS}

Nous remercions MM. Menissier (Station de Génétique quantitative et appliquée, C. N. R. Z., 78 Jouy en Josas) et Ricard (Station expérimentale d'Aviculture du Magneraud, 7 Surgères) de leurs critiques et suggestions à la lecture de ce manuscrit.

\section{SUMMARY}

\section{COMB OR WATTLE SIZE OF IO WEEKS OLD COCKEREIS AS AN INDICATOR OF SEXUAL MATURITY IN SELECTION FOR EGG PRODUCTION}

In our population, the mean wattle length of brothers at io weeks presents an appreciable negative correlation with age at first egg of their full sisters, and a slight positive correlation with their fall and winter egg laying. The genetic correlations have the same signs, and the heritability of wattle length is relatively high. This suggests a possible use of this trait as individual selection criterion on cockerels for their genetic potential for age at sexual maturity.

\section{RÉFÉRENCES BIBLIOGRAPHIQUES}

Galpern I., Bongaiev G. I., I966. (Appréciation précoce des qualités de reproduction et de production). Publ. minist. Agric. U.R. S. S., Moscou, Édit. Colos.

Goodwin K., Cole K., Hutt F. B., Rasmusen B. A., 1955. Endocrine relationships in males of a relatively infertile strain of white Leghorn fowls. Endocrinology, 57, 5 19-526.

Kempthorne O., 1957. An introduction to genetic statistics. J. Wiley and sons, New York.

Kinney T. B. Jr, Shoffner R. N., I965. Heritability estimates and genetic correlations among several traits in a meat type poultry population. Poult. Sci., 44, ro20-1032.

MÉrat P., r967. Contribution à l'étude de la valeur sélective associée à quelques gènes chez la Poule domestique. I. Différences quantitatives liées au génotype individuel. Ann. Biol. anim. Bioch. Biophys., 7, 79-104.

Pasvogel M. W., 1952 . Correlation between the egg laying ability of pullets and certain hormonally controlled characteristics of the full brothers. $\mathrm{Ph}$. D. Thesis, University of Illinois.

Pasvogel M. W., Nalbandov A. V., Morton H. W., r95r. Relationship between comb growth in the male and egg production in the sibs. Poult. Sci., 30, 926 (abstr.). 